\section{Comparison of matching and drawing in the perception of shape at various intelligence levels*}

\author{
HERSCHEL W. LEIBOWITZ $\dagger$ and ELIZABETH J. SACCA $\dagger \dagger$ \\ The Pennsylvania State University, University Park, Pennsylvania 16802
}

The tendency toward shape constancy was investigated using both the traditional matching technique of Thouless and free-hand drawing of the stimulus object. Data were obtained for college students and for institutionalized feebleminded Ss at three levels of intelligence. The previous observation that $\mathrm{Ss}$ of higher intelligence level produce a lower tendency toward shape constancy, as measured by the matching method, was confirmed. With the drawing method, the same trend was observed but the differences among groups were exaggerated. The sensitivity of measures of perceived shape to experimental as well as nonexperimental variables is discussed.

It is well known that quantitative values of matched shape are strongly influenced by the instructions given to $\mathrm{S}$. Klimpfinger (1933) has shown that "synthetic" instructions, which emphasize the true shape of the test-object, resulted in a greater tendency toward constancy than did "analytic" instructions, which emphasize the projected shape. ${ }^{1}$ The effect of instructions or assumed instructions on matched shape has been confirmed by a number of subsequent experiments (Epstein \& Park, 1963; Landauer, 1964, 1969; Kaess, 1970).

While shape matching, probably because of convenience of quantification, has been the method of choice in the experimental psychology literature, it is not the only possible method of studying shape perception. Thouless (1931), in his pioneer studies of shape constancy, initially used the drawing method but later abandoned it in favor of shape matching. He stated that the matching method was "better" than drawing but did not provide evidence to support this point of view. In view of the sensitivity of matched shape to instructions, as well as to spontaneously assumed attitudes (Joyson \& Newson, 1962), it is of interest to compare the values obtained from shape matching and drawing since the two tasks involve both different instructions and modes of response. To this end, the present study investigates matching and drawing of round objects at two angles of inclination. Since

Supported by Grant MH-08061 from tho National Institute of Mental Ilealth, Public Health Service. The authors wish to express their appreciation to the staft of the Selinsgrove State School and Hospial for their cooperation and 10 Dr. Peter Kuiser, Robert T. Hemnessy, and John Cegalis lor help in the preparation of the manuscript.

Address: Psychology Butdiny, The Pennsylvania State University, University Park. Pennsylvania 16802.

$\dagger \dagger$ Now at Department of Art, Ball State University, Muncic, Indiana 47306. it has already been shown that the tendency toward shape constancy for a matching task varies as a function of intelligence level (Leibowitz, Waskow, Loeffler, \& Glaser, 1959), it is of interest to determine if this same relationship also holds for the drawing technique.

\section{METHOD}

\section{Apparatus}

The stimuli consisted of two white (reflectance $90 \%$ ) cylinders, each 7 in. long and $4 \frac{1}{4} \mathrm{in}$. in diam, resting on a gray surface and viewed against a similar gray (reflectance 30\%) background. One cylinder was placed in a vertical position, and the other, $7 \mathrm{in}$. to the right, horizontally on its side. Two angles of inclination of the surface on which the cylinders rested were used, horizontal or $30 \mathrm{deg}$ from the horizontal. When the surface was horizontal, the axis ratio of the projected image at $S$ 's eye (ratio of minor for both cylinders. As a result of the orientation of the cylinders, the minor axis of the vertical cylinder was projected vertically in the frontal plane, while that for the cylinder lying on its side was horizontal. As the surface was inclined $30 \mathrm{deg}$, the foreshortening of the minor axis of the vertical cylinder was decreased such that the ratio became .77 . The other cylinder, however, was rotated about the vertical axis of its nearer end to compensate for foreshortening so that its axis ratio remained at $\mathbf{4 2}$.

The S's head was at all times $71.6 \mathrm{in}$. from and $32 \mathrm{in}$. above the top of the vertical cylinder. When viewing the cylinders, S's eyes were $51 \mathrm{in}$. above the floor. Observation was binocular.

\section{Procedure}

The matching involved the use of 17 solid white ellipses mounted individually on $8 \times 10 \mathrm{in}$. gray cards. These ellipses were constructed by photographing a white to major axis of the cross section) was .42 circular $d$ isk at various angles of inclination, chosen so that the minor axis of the imaged ellipses varied from $1-1 / 8$ to 4-1/4 in. in equal steps.

Each $\mathrm{S}$ matched and drew both cylinders at the two angles of inclination. The $S$ was asked to draw the object with a pencil on white paper mounted on a clipboard. For the matching task, the Ss were shown the comparison stimuli individually, the 17 solid white ellipses, at a distance of $20 \mathrm{in}$. and were asked, "Which looks rounder?" i.e., the shape on the card or the top of the cylinder. $S$ indicated his choice by pointing to it. The order of presentation of the comparison ellipses and the other experimental variables were randomized. The comparison matching value was obtained by interpolation from the series as the value at which the $S$ 's responses changed. The response of "same" was accepted when offered. For the drawing procedure, the ratio of minor to major axis was determined by direct measurement.

Subjects

The performance of $\mathbf{S s}$ representing four levels of intelligence was tested. The highest group consisted of 36 college students enrolled in introductory psychology courses. The remaining $33 \mathrm{Ss}$ were mentally retarded students, of the same chronological age as the college students, resident in a state institution for the retarded. These were divided into three groups of 11 members each with IQs ranging from 81 to 70,69 to 60 , and 59 and below.

\section{RESULTS}

The data are plotted in Fig. 1 as the mean minor/major axis ratio for matching and for drawing with intelligence level as a parameter. Considering first the data for matching, it will be noted that data for the vertical cylinder at the various intelligence levels cluster together. For the horizontal cylinder, there is greater separation among the various levels, with the tendency towards constancy inversely related to intelligence. For the drawing task, intelligence-level differences arc exaggerated, with the more intelligent Ss tending to produce lower values. The Ss with the lowest intelligence level produced the highest matches under all four conditions, while the most intelligent Ss produced the lowest matches for three of the four conditions. Intelligence level $(F=21.7, \mathrm{df}=3 / 65)$, task, i.e., matching vs drawing $(F=13.9, \mathrm{df}=1 / 65)$, and the interaction between intelligence level and task $(F=10.9, \mathrm{df}=3 / 65)$ are all significant beyond the .01 level of confidence.

\section{DISCUSSION}

The data of the present study confirm 


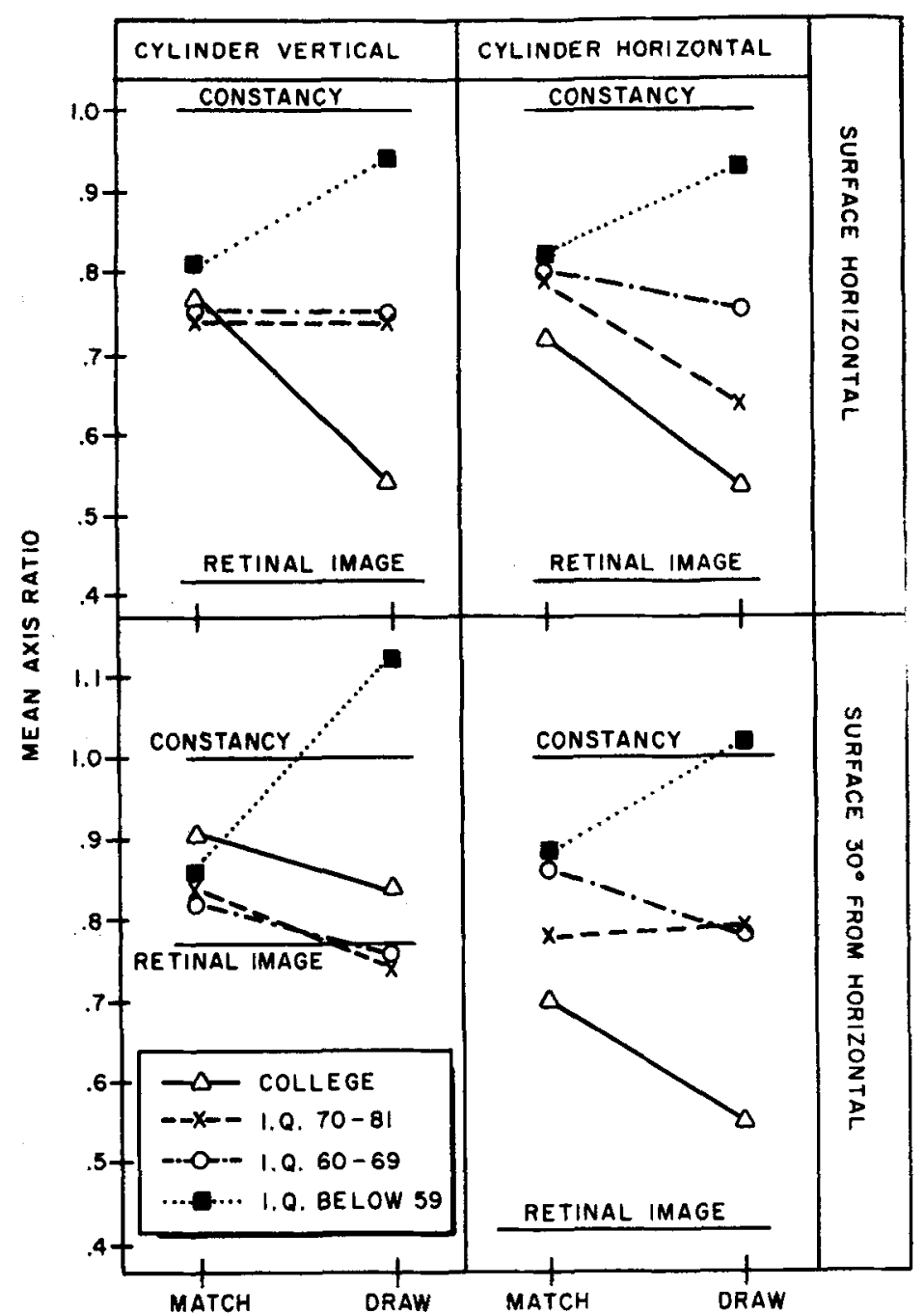

Fig. 1. Mean axis ratio for matching and drawing a vertical and horizontal cylinder at two angles of orientation.

the previous observation that the tendency toward shape constancy is inversely related to $S$ 's intelligence level (Leibowitz, Waskow, Loeffler, \& Glaser, 1959). These functions may be interpreted either as (1) true perceptual differences or (2) that the shapes were seen the same but the interpretations of the instructions were responsible for the observed differences. We strongly favor the latter interpretation and feel that the most likely basis for the present group differences is to be found in the varying reactions to the experimental situation by the various groups.

It is assumed that the more intelligent Ss adopt a more analytic attitude to the experimental task, while the less intelligent Ss adopt a more synthetic attitude. Although the instructions are objectively identical, the interpretation of the task depends upon the S's intelligence level. ${ }^{2}$ This position implies that the differences observed here represent an extension of the empirical observation that instructions to true shape of the object in question than are the more intelligent Ss.

The present results demonstrate that the quantitative evaluation of shape perception is extremely sensitive to both implicit and explicit conditions, not all of which are under the control of E. Instructions can be interpreted differently, depending upon S's reaction to the experimental situation. In addition, the mode of response, e.g., drawing vs matching, is shown here to be a major variable which interacts with implicit and, most probably, with explicit instructions as well. In view of these findings, one may reasonably ask which is the true measure of shape constancy. Our answer would be to point out that perceived shape is a loosely defined term, which encompasses both experimental and nonexperimental situations. Outside of the laboratory, the impression of shape is only one of many stimulus attributes of interest. In contrast, in the laboratory situation by virtue of the specific task, and the instructions to match or to draw, the shape dimension is emphasized to the exclusion of other stimulus attributes. Operationally, different procedures are involved in the experimental and nonexperimental situations, the former involving matching or drawing, and the second a phenomenal impression of shape. Even within the experimental paradigm, different explicit instructions, differences in assumed implicit instructions, and the various modes of response all represent different procedures and operationally distinct measures of shape perception. Furthermore, these different procedures are scen to produce different quantitative values of perceived shape. In view of this, we would suggest that the question of the "true" measure of perceived shape is meaningless. Measures that purport to reflect perceptions are influenced, to a great extent, by response biases. Although perception and response can be differentiated conceptually, it is difficult to separate them in the experimental context. For this reason, the quantitative evaluation of shape perception per se is not feasible. Rather, one is limited to investigation of the relation between experimental conditions and observer responses which are influenced, as shown in the present study, by the intelligence level of the $S$ and the mode of his response.

\section{REFERENCES}

structured matching situation. These results suggest that drawing is a morc sensitive method for studying individual differences in shape constancy. These data are relevant not only to the study of shape constancy, but also to art education. In a drawing task, less intelligent Ss are more likely to respond initially in terms of the
IPSTEIN, W., \& PARK, J. N. Shape constancy:
Functional relationships and theoreticul formujatjons. Psychological Bulletin, 1963,60, 265-288.

JOYSON, R. B., \& NEWSON, L. J. The perception of shape als a function of inclination. British Joumal of Psychology, 1962,53, 1-15. 
KAESS, D. W. Form constancy and the perceptual tasks: A developmental study. Journal of Experimental Psychology, 1970, $83,465-471$.

KLIMPFINGER, S. Über den Einfluss von intentionaler Einstellung und Übung auf die Gestaltkonstanz. Archiv für die gesamte Psychologie, 193, 88, 551-598.

LANDAUER, A. A. The effect of viewing conditions and instructions on shape judgments. British Journal of Psychology, $1964,55,49-57$.
LANDAUER, A. A. Influence of instructions on judgments of unfamilias shapes. Journal of Experimental Psychology, 1969, 79, 129-132. LEIBOWITZ, H. W., WASKOW, I., LOEFFLER, N., \& GLASER, F. Intelligence level as a variable in the perception of shape. Quarterly Journal of Experimental Psychology, 1959. $11,108-112$.

ORNE, M. The nature of hypnosis: Artifact and essence. Journal of Abnormal \& Social Psychology, 1959, 58, 277-299.

THOULESS, R. H. Phenomenal regression to the "real" object. I, II. British Journal of Psychology, 1931, 21, 339-359; 22, 1-30.

\section{NOTES}

1. The Brunswick ratios or pereent constancy were between .60 and .90 tor synthetic instructions and between .18 and .33 for analytic instructions.

2. This phenomenon has been evaluated in a broader context under the heading of "demand characteristics" by Orne (1959).

(Accepted for publication A ugust 28, 1970.) 